\title{
Management and GGBN publishing of tissue and DNA extract data through Specify software
}

\author{
Andrew C Bentley ₹ \\ ‡ University of Kansas, Lawrence, KS, United States of America
}

Corresponding author: Andrew C Bentley (abentley@ku.edu)

Received: 17 May 2018 | Published: 05 Jul 2018

Citation: Bentley A (2018) Management and GGBN publishing of tissue and DNA extract data through Specify software. Biodiversity Information Science and Standards 2: e26749. https://doi.org/10.3897/biss.2.26749

\begin{abstract}
Due to the recent establishment of the Global Genome Biodiversity Network (GGBN) data portal, we have extended Specify collections management software (http:// www.sustain.specifysoftware.org/) to more effectively manage, publish, and integrate tissue and DNA extract data by adding support for the GGBN data schema. Specify's database design now includes a number of data fields and tables proscribed in GGBN standard vocabularies. We also realigned some of the underlying table relationships to address the needs of specimen curation and collection transactions for extract and tissue samples. Specify now also supports "Next Generation" sequencing metadata with fields to record NCBI SRA ID numbers for web-linking tissue and extract metadata to entries in the NCBI SRA databases.

With the ongoing evolution of the TDWG Darwin Core (DwC) standard for specimen data exchange, we generalized Specify 7's data publishing capabilities to export collections data to any DwC or other standards-based, exchange schema. This generic, external schema mapping capability enables Specify collections to design and map data packages to integrate their data with any community aggregator or collaborative project database based on Darwin Core or other community standard-based format. The development of these versatile new integration capabilities was in collaboration with, and through financial support from GGBN. This talk will highlight these changes in the context of delivery of museum tissue and extract data records to the GGBN data portal for aggregation.
\end{abstract}




\section{Keywords}

Specify Software, GGBN, tissue, extract, DNA, database design

\section{Presenting author}

Andrew C Bentley

\section{Funding program}

DBI - ADVANCES IN BIO INFORMATICS

\section{Grant title}

ABI Sustaining: Supporting Biological Collections Computing with Specify 